\title{
Communication System Based on Periodic Nonlinear Fourier Transform with Exact Inverse Transformation
}

\author{
Morteza Kamalian, Anastasiia Vasylchenkova, Jaroslaw Prilepsky, Dmitry Shepelsky, Sergei Turitsyn
}

Aston Institute of Photonic Technologies, Aston University, Birmingham, UK. kamaliam@aston.ac.uk

\begin{abstract}
By performing the exact inverse transformation, a periodic solution to channel model is constructed and used in an NFT-based communication system. The achievable mutual information is calculated using the non-uniform probability distribution for transmitted symbols for different link lengths.
\end{abstract}

\section{Introduction}

Nonlinear Fourier transform (NFT) has been shown to be a highly promising tool in overcoming the detrimental impact arising due to the Kerr nonlinearity in fibre-optic systems ${ }^{1,2}$. However, the NFT-based transmission concept still has some unresolved problems ${ }^{2}$. One of the challenges of implementing the NFT in Optical communications is to control the duration of modulated signal at the transmitter (Tx): we wish to make it as short as possible to have a larger net data rate and system's spectral efficiency $(\mathrm{SE})^{3,4}$. That duration can be controlled by, e.g., using the recent NFT-based modulation technique named the $b$ coefficient modulation ${ }^{1}$, resulting in optical signals having a finite controllable duration. Another solution to this problem is to find a periodic solution to the channel model (the nonlinear Schödinger equation, NLSE, for our current work) with the required duration, i.e. with a predefined signal period. So we can use the signals extended by a cyclic prefix, similar to OFDM. This approach was investigated in ${ }^{5,6}$, where due to the challenging complexity of exact inverse transformation, an approximation technique was adopted to construct a signal at Tx. To this aim, the random data is mapped on the separation of complex points in the so-called main spectrum of a perturbed plane wave, given this separation is small enough ${ }^{7,8}$. This limitation constrains the class of available signals to a set with a confined bandwidth and signal power, and renders a QAM constellations with a small minimum distance. The latter leads to a larger bit error rate due to the presence of numerical error and optical noise. In this work, for the first time, we construct an exact periodic solution to the NLSE with arbitrary period, which has enough degrees of freedom to provide a truly 2-dimensional constellation.

\section{Nonlinear spectrum for periodic signals}

NFT provides an infinite number of solutions to NLSE, parametrised through the associated non- linear spectra (NS). For the periodic NFT (PNFT), the NS consists of auxiliary and main spectrum (eigenvalues) parts ${ }^{7}$. For a periodic solution containing $N$ eigenvalues in its NS, there are $4 N$ degrees of freedom available for modulation: $2 \mathrm{~N}$ from the complex eigenvalues positions and $2 \mathrm{~N}$ attributed to the auxiliary spectrum. In this work we use for the modulation only the main spectrum part of NS. Since it is necessary to have at least two independent parameters to make up a QAM constellation (after determining the signal period), we work with signals having only two complex eigenvalues in their NS. Such signals can be constructed considering the associated Zakharov-Shabat system of equations ${ }^{5,7}$, constituting the forward PNFT. Then, the periodic NLSE solutions can be generated through solving a set of involved differential equations describing the evolution of auxiliary spectrum ${ }^{7,9}$. Solving such a complicated system is not feasible for communication purposes even for the simplest case. Therefore, a change of variable, called the Abel map, is usually implemented to turn this complicated evolution into a linear relations in time and distance ${ }^{7}$. In this way, an exact periodic solution to the NLSE can be obtained. Then to construct a periodic solution to the NLSE we need to evaluate a multi-dimensional Fourier series called the Riemann theta function ${ }^{7,9}$. This approach again demands a considerable amount of computational resource, which makes it hardly possible to implement in communications. However, assuming

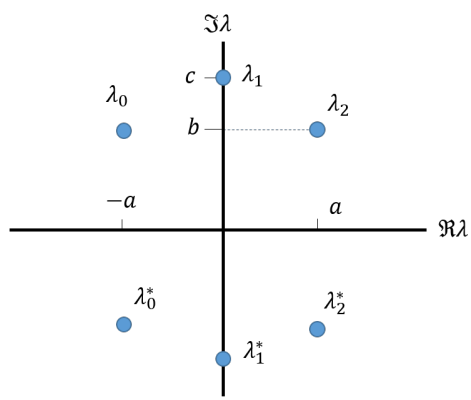

Fig. 1: The nonlinear spectrum of a periodic solution with circles representing the discrete spectrum. 


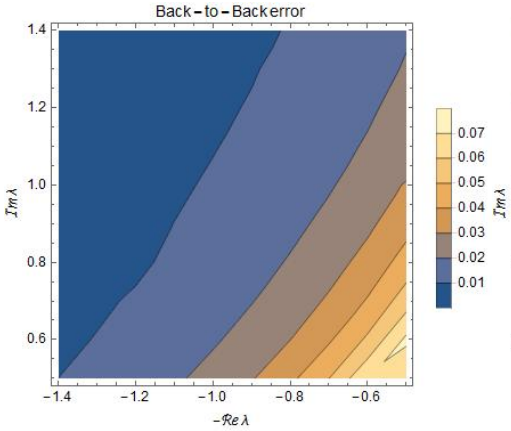

Fig. 2: B2B error for different locations

Fig. 2: B2B error for different locations $\quad$ Fig. 3: Signal power for different
of the eigenvalue with $\Re \lambda=a, \Im \lambda=b$ locations of the eigenvalue with $\Re \lambda=a$, and $a, b$ defined in Fig. 1 .

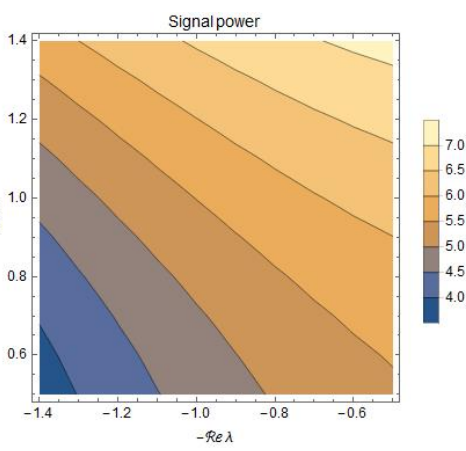
$\Im \lambda=b$ and $a, b$ defined in Fig. 1 .

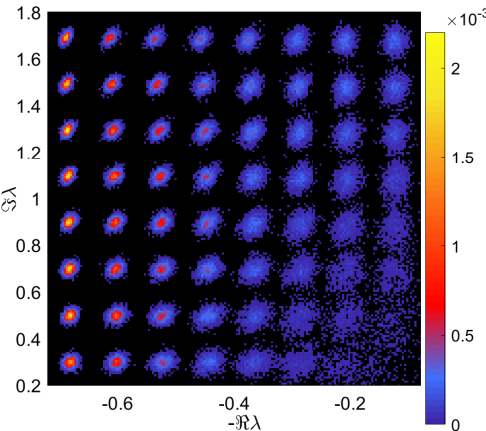

Fig. 4: The received constellation at $z=1120 \mathrm{~km}$ for a 64-QAM signal with power $P=-2.72 \mathrm{dBm}$. some symmetry in the main spectrum, one can reduce this function into simpler one-dimensional elliptic functions ${ }^{10}$. The main spectrum example allowing such a reduction is shown in Fig. 1, with three pairs of complex conjugate eigenvalues $\{-a+b i, c i, a+b i\}^{10}$. Parameters $a$ and $b$ carry the data and can be recast as a QAM symbol. Parameter $c$ is then determined by the choice of $a$ and $b$ and by the desired solution period value.

\section{Probabilistic shaping}

The numerical accuracy of performing the inverse PNFT (as explained above) and direct PNFT ${ }^{11}$ depends on the number of time samples, signal power, and the particular location of eigenvalues. The back-to-back (B2B) error without any random noise shows the dependence of the numerical accuracy on the location of eigenvalues, Fig. 2. This error is defined as the Euclidean distance between the transmitted and received eigenvalue. Simulation results show that the B2B error and the error at the receiver after a noisy transmission have similar patterns with regard to their dependence on the eigenvalues location, which yet again, verifies that the main contributor to the overall error is the numerical one for the PNFT. Here, a Gaussian mixture model of the channel is considered, where the conditional probability $P(\hat{\lambda})$ of the received eigenvalue, $\hat{\lambda}$, on the transmitted one, $\lambda$, is assumed to be Gaussian with a $\lambda$-dependent variance $\sigma_{\lambda}$. Therefore we have ${ }^{3,12}$ :

$$
P(\hat{\lambda})=\sum_{\lambda \in \Lambda} P_{\lambda} \mathcal{N}\left(\hat{\lambda} ; \lambda, \sigma_{\lambda}\right)
$$

with $\Lambda$ being the main spectrum set, $P_{\lambda}$ the probability of sending $\lambda$ and $\mathcal{N}\left(\hat{\lambda} ; \lambda, \sigma_{\lambda}\right)$ denotes a Gaussian distribution for random variable $\hat{\lambda}$ with mean $\lambda$ and variance $\sigma_{\lambda}$. The standard deviation, $\sigma_{\lambda}$, is estimated from the received points. As mentioned before, $\sigma_{\lambda}$ has the same pattern as the B2B error shown in Fig. 2 which in turn depicts a considerable variation between different points. This in fact, suggests that we can apply a non-uniform probability distribution for the transmitted symbols, in which the constellation points that contribute more to the overall error are used less frequently. On the one hand, our excluding the eigenvalues with high contribution to EVM leads to a better $Q^{2}$-factor, but reduces the mutual information (MI). Hence, keeping the constellation size, $M$, and the main spectrum, $\Lambda$, fixed, we optimise $P_{\lambda}$ in (1) to have the maximum Ml, $I(\hat{\lambda}, \lambda)$. This is numerically done for each signal power and link length, and for each value of parameters the EVM is used to calculate $\sigma_{\lambda}$.

\section{Simulation results}

At the Tx the bit stream is mapped onto the QAM constellation following a non-uniform probability mass function which is obtained from the $\mathrm{B} 2 \mathrm{~B}$ error distribution or the receiver EVM. In the first set of simulations, from the chosen eigenvalues set we have the signal power $P=-2.7 \mathrm{dBm}$. According to Fig. 3, $P$ can be controlled through changing the location of eigenvalues, for example by increasing $\Im \lambda$ while keeping the signal bandwidth fixed. Considering the signal power (Fig. 3 in arbitrary units for different locations of the eigenvalues) along with the B2B error (Fig. 2), one can realise that the $\mathrm{B} 2 \mathrm{~B}$ error does not directly depend on the signal power. Therefore, the average launch power does not vary with uniform or nonuniform symbol allocation if the distribution is determined with respect to the B2B error. According to each symbol, a periodic signal is produced following the steps in ${ }^{10}$ and cyclically extended to the size of chromatic dispersion memory. The burst of symbols then is formed by packing several signals and sent to the link with lumped amplification and a fibre with loss coefficient $\alpha=0.2$ $\mathrm{dB} / \mathrm{km}$, dispersion parameter $\beta=-20 \mathrm{ps}^{2} / \mathrm{km}$, and Kerr nonlinearity parameter $\gamma=1.3 / \mathrm{W} / \mathrm{km}$.

At the $R x$, the main spectrum of the signal 

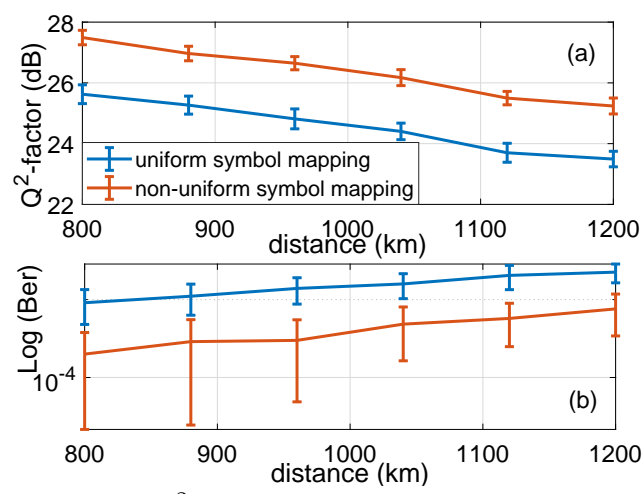

Fig. 5: a) the $Q^{2}$-factor calculated from the $E V M$, and $b$ ) directly calculated BER against distance for 64-QAM with uniform and non-uniform transmitter symbol probability with average $P=-2.72 \mathrm{dBm}$ signal power.

is calculated by the PNFT ${ }^{11}$ and the transmitted data are retrieved. A simple blind phase estimation is used to equalise some distortions which are mainly coming from signal broadening and insufficient cyclic prefix. The length of cyclic prefix is fixed to the signal broadening after $1000 \mathrm{~km}$.

Fig. 5 shows the $Q^{2}$-factor calculated from the EVM at the receiver and the BER (directly calculated from counting the mismatches between the Tx and Rx symbol streams) vs different propagation distances. From this figure one can compare the results when a uniform and non-uniform distribution for the transmitted symbols is considered. These result are calculated for 200 transmissions of symbol bursts, each having the length $12 \times M$, where $M$ is the size of constellation. As it can be seen from Fig. 5, a considerable improvement of $2 \mathrm{~dB}$ in the $\mathrm{Q}^{2}$-factor can be earned by the probabilistic shaping of constellation. However, a more informative figure is the maximum achievable error-free MI or SE for various link lengths. Thanks to the used exact inverse PNFT, a full control over the signal's power and time duration makes it possible to optimise the signal in terms of its parameters, and to find the maximum SE. The achievable $\mathrm{Ml}$ in bits per symbol for a discretemodulation NFT system with one eigenvalue is presented in Fig. 6. It is worth noting that these results are to be compared with other discrete NFT communication systems such as eigenvalue communication in which the throughput of the system is smaller when compared to a continuous spectrum modulation ${ }^{3}$.

\section{Conclusion}

For the first time, an exact inverse transformation for PNFT is performed and the resulted signal is used in a fibre-optic communication system. Considering the B2B error caused by the numerical routines inaccuracy, a probabilistic shaping for a

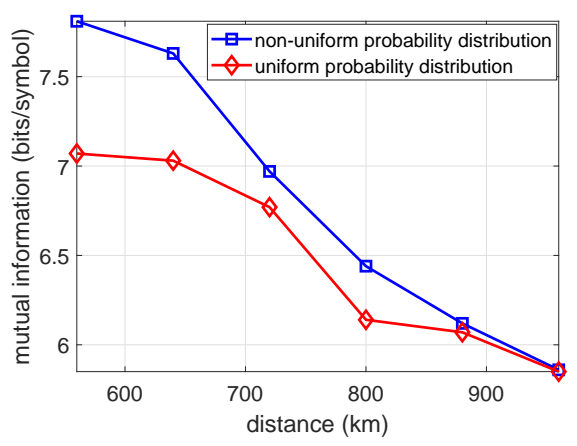

Fig. 6: Achievable mutual information versus distance for a signal with average power of $P=-2.72 \mathrm{dBm}$ with uniform and non-uniform probability distribution of the transmitted symbols.

Gaussian mixed model is applied, leading to a 2$\mathrm{dB}$ gain in the $\mathrm{Q}^{2}$-factor and improvement in the achievable MI.

\section{Acknowledgements}

This work was partially supported by the Leverhulme Project RPG-2018-063.

\section{References}

[1] S. Le et al, "100 Gbps b-modulated Nonlinear Frequency Division Multiplexed Transmission," OFC (2018).

[2] S. Turitsyn et al, "Nonlinear Fourier transform for optical data processing and transmission: advances and perspectives," Optica 4, 307-322 (2017).

[3] H. Bülow et al, "Modulation on Discrete Nonlinear Spectrum: Perturbation Sensitivity and Achievable Rates," IEEE Photonic Tech. Lett. 30, 423-426 (2018).

[4] X. Yangzhang et al, "Impact of Perturbations on Nonlinear Frequency-Division Multiplexing," J. Lightwave Technol. 36, 485-494 (2018).

[5] M. Kamalian et al, "Periodic nonlinear Fourier transform for fiber-optic communications, Part II: eigenvalue communication," Opt. Express, vol. 24, 2016.

[6] M. Kamalian et al, "Optical communication based on the periodic nonlinear Fourier transform signal processing," IEEE 6th International Conference on Photonics (ICP), Kuching, Malaysia, pp. 1-3, 2016.

[7] M. Kamalian et al, "Periodic nonlinear Fourier transform for fiber-optic communications, Part I: theory and numerical methods," Opt. Express, vol. 24, 2016.

[8] M. Kamalian et al, "Spectral efficiency estimation in periodic nonlinear Fourier transform based communication systems," OFC, paper Th2A.54, 2017.

[9] A. Osborne, "The random and deterministic dynamics of 'rogue waves' in unidirectional, deep-water wave trains," Mar. Struct. 14, 275-293 (2001).

[10] A. Smirnov, "Periodic Two-Phase "Rogue Waves", Math. Notes, 94, 871-883 (2013).

[11] S. Wahls, H. Poor, "Fast numerical nonlinear Fourier transforms," IEEE T. Inform. Theory, 61 (2015).

[12] T. Fehenberger et al, "On probabilistic shaping of quadrature amplitude modulation for the nonlinear fiber channel." J. Lightwave Technol. 34, 5063-5073 (2016). 